\title{
Use of Flattening Filter Free Photon Beams for Off-axis Targets in Conformal Arc Stereotactic Body Radiation Therapy
}

\author{
Ashley Smith*, Siyong $\mathrm{Kim}^{\dagger}$, Christopher Serago*, Kathleen Hintenlang*, Stephen Ko*, Laura Vallow*, \\ Jennifer Peterson*, David Hintenlang ${ }^{\ddagger}$, Michael Heckman ${ }^{\$}$, Steven Buskirk* \\ *Division of Radiation Oncology, Mayo Clinic, 4500 San Pablo Road South, Jacksonville, FL, 32224, \\ ${ }^{\dagger}$ Department of Radiation Oncology, Virginia Commonwealth University, 401 College St, Richmond, VA \\ 23284, ${ }^{\ddagger} \mathrm{J}$ Crayton Pruitt Family Department of Biomedical Engineering, University of Florida, 1275 Center \\ Drive, Gainesville, FL 32611, `Division of Biomedical Statistics and Informatics, Mayo Clinic, 4500 San Pablo \\ Road South, Jacksonville, FL, 32224
}

\begin{abstract}
Dynamic conformal arc therapy (DCAT) and flattening-filter-free (FFF) beams are commonly adopted for efficient conformal dose delivery in stereotactic body radiation therapy (SBRT). Off-axis geometry (OAG) may be necessary to obtain full gantry rotation without collision, which has been shown to be beneficial for peripheral targets using flattened beams. In this study dose distributions in OAG using FFF were evaluated and the effect of mechanical rotation induced uncertainty was investigated. For the lateral target, OAG evaluation, sphere targets (2, 4, and $6 \mathrm{~cm}$ diameter) were placed at three locations (central axis, $3 \mathrm{~cm}$ off-axis, and $6 \mathrm{~cm}$ off-axis) in a representative patient CT set. For each target, DCAT plans under the same objective were obtained for $6 \mathrm{X}$, 6FFF, 10X, and 10FFF. The parameters used to evaluate the quality of the plans were homogeneity index (HI), conformality indices $(\mathrm{Cl})$, and beam on time (BOT). Next, the mechanical rotation induced uncertainty was evaluated using five SBRT patient plans that were randomly selected from a group of patients with laterally located tumors. For each of the five cases, a plan was generated using OAG and CAG with the same prescription and coverage. Each was replanned to account for one degree collimator/couch rotation errors during delivery. Prescription isodose coverage, $\mathrm{Cl}$, and lung dose were evaluated. $\mathrm{HI}$ and $\mathrm{Cl}$ values for the lateral target, OAG evaluation were similar for flattened and unflattened beams; however, 6FFF provided slightly better values than 10FFF in OAG. For all plans the $\mathrm{HI}$ and $\mathrm{Cl}$ were acceptable with the maximum difference between flattened and unflattend beams being 0.1 . FFF beams showed better conformality than flattened beams for low doses and small targets. Variation due to rotational error for isodose coverage, $\mathrm{Cl}$, and lung dose was generally smaller for CAG compared to OAG, with some of these comparisons reaching statistical significance. However, the variations in dose distributions for either treatment technique were small and may not be clinically significant. FFF beams showed acceptable dose distributions in OAG. Although 10FFF provides more dramatic BOT reduction, it generally provides less favorable dosimetric indices compared to 6FFF in OAG. Mechanical uncertainty in collimator and couch rotation had an increased effect for OAG compared to CAG; however, the variations in dose distributions for either treatment technique were minimal.
\end{abstract}

Key Words: Flattening-filter-free, Lateral targets, Conformal arc, SBRT

\section{Introduction}

Received 18 November 2014, Revised 23 November 2014, Accepted 14 December 2014

Correspondence: Ashley Smith (smith.ashley@mayo.edu) Tel: 1-904-953-7903, Fax: 1-904-953-1010

(C) This is an Open-Access article distributed under the terms of the Creative Commons Attribution Non-Commercial License (http://creativecommons.org/licenses/by-nc/3.0) which permits unrestricted non-commercial use, distribution, and reproduction in any medium, provided the original work is properly cited.
Dynamic conformal arc therapy (DCAT) in full co-planar rotation is commonly adopted for efficient conformal dose delivery in stereotactic body radiation therapy (SBRT). ${ }^{1,2)}$ Flatteningfilter-free (FFF) beams can increase delivery efficiency by employing a high-dose rate that greatly reduces the beam on time (BOT) ${ }^{3-8)}$ This makes them useful for treatments requiring a 
high dose per fraction, such as SBRT. The increased efficiency of FFF beams can also aid in respiratory motion management, including the use of voluntary breath hold techniques, by enabling entire beam delivery within a short number of breath holds. ${ }^{7)}$ DCAT is currently being used with FFF beams to treat SBRT to further increase delivery efficiency.

For laterally located targets, which are commonly seen in lung and liver SBRT, it is often necessary to use off-axis geometry (OAG) to obtain full rotation of the gantry without collision of the gantry head with either the patient or the treatment couch. OAG occurs when the isocenter is positioned at patient midline; however, the target is located laterally off-axis (Fig. 1). Central axis geometry (CAG) occurs when the isocenter is located at the center of the target, regardless of its position within the patient. The use of a modified DCAT technique, which utilizes a midline isocenter, has been shown to be useful for the treatment of peripheral lung tumors using SBRT. ${ }^{9,10)}$ These studies have demonstrated that the use of a modified DCAT technique results in enhanced PTV coverage, improved conformality, reduced BOT, and meets the requirements of RTOG protocols. We believe it is important to study this technique with FFF beams, which has not been done. FFF beams employ a dose rate much higher than the standard dose rate by removing the flattening filter from the path of the beam, which causes a significantly non-uniform incident fluence profile. Because the off-axis profile of an FFF beam is not uniform, we investigated whether FFF beams can provide acceptable dose distributions in OAG.

While the incident fluence profile of FFF beams is significantly non-uniform, there are several advantages of FFF beams compared to conventional beams that could influence dosimetry for lateral lesions. FFF beams have less variation of off-axis beam hardening due to the removal of the flattening filter from the path of the beam. FFF also has less photon-head scatter due to removing the flattening filter, which results in less field size dependence, as well as less leakage outside of beam collimation. The peripheral dose far from the field edge $(15 \sim 20 \mathrm{~cm})$ is influenced by the treatment head leakage, which has been shown to be reduced by $52 \%$ for 6 $\mathrm{MV}$ and $65 \%$ for $10 \mathrm{MV}$ unflattened beams. ${ }^{11)}$ The penumbra size and multi-leaf collimator leakage are also reduced for FFF beams. $^{12,13)}$

While these advantages may make it desirable to use FFF beams, it is necessary to quantitatively characterize methods for dose planning and delivery for lateral lesions. Parameters associated with implementing FFF treatments were systematically investigated in this study. Additionally, due to the distance between the axis of rotation and the target for OAG, rotational error will have an increased effect on the dose distribution compared with CAG. If OAG is used, there is the possibility of increased delivery uncertainty due to mechanical errors associated with collimator or couch rotation. We investigated the effect of collimator and couch mechanical error with an error uncertainty analysis to examine whether the delivery uncertainty is acceptable.

\section{Materials and Methods}

\section{Lateral target, off-axis geometry evaluation}

A total of nine sphere targets were placed on a representa-

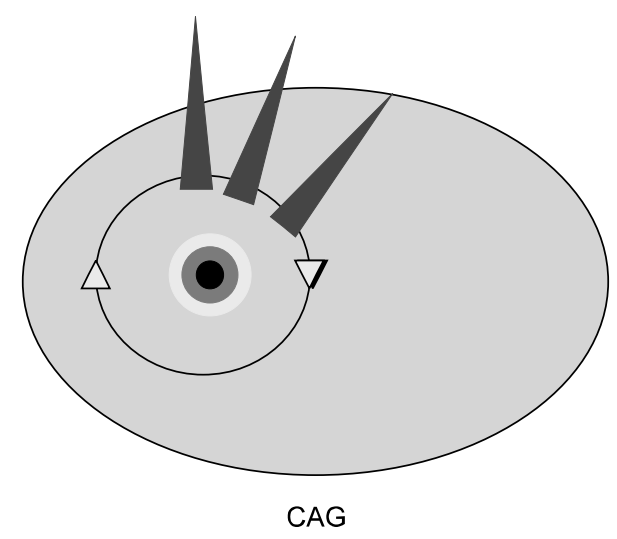

CAG

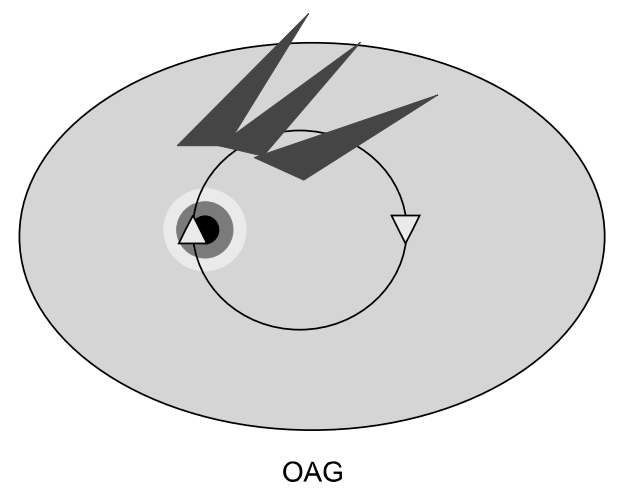

Fig. 1. Central axis geometry (CAG) and off-axis geometry (OAG) for a laterally located target. 
tive computed tomography (CT) image set selected from lung SBRT patients treated at Mayo Clinic in Florida (GE LightSpeed RT CT Scanner; GE Healthcare, Waukesha, Wisconsin) to represent a typical SBRT patient. Lung SBRT patients are simulated supine with arms down in a whole body Vac-Q-Fix Cushion ${ }^{\mathrm{TM}}$ (Qfix, Avondale, PA) and abdominal compression using a balloon with a pressure gauge. ${ }^{14,15)}$ The CT image set was transferred to a Pinnacle treatment planning system (Pinnacle Version 9 software; Philips Medical Systems, N.A., Bothell, Washington). Fig. 2 shows the nine sphere targets have three different sizes $(2,4$, and $6 \mathrm{~cm}$ diameter) and are placed at three different locations (center of the patient, 3 $\mathrm{cm}$ lateral, and $6 \mathrm{~cm}$ lateral). The isocenter was located at the center of the patient for all targets. For each target, DCAT plans were obtained using four different beams $(6 \mathrm{X}, 6 \mathrm{FFF}$, 10X, and 10FFF) for a Varian TrueBeam LINAC (Varian TrueBeam; Varian Medical Systems, Palo Alto, California). Plans were normalized such that $95 \%$ of the target volume received the prescription dose. For all plans, two conformality indices (CI), CI-100 (prescription isodose volume to target volume) and CI-50 (50\% prescription isodose volume to target volume) were calculated. The minimum possible CI-100 value is 0.95 with exact $95 \%$ coverage. Homogeneity index (HI) was also calculated for each plan using the definition commonly used for radiosurgery (ratio of max dose to prescription dose). ${ }^{16,17)}$ BOT was estimated based on planned monitor units (MU) and the maximum dose rate available in each beam

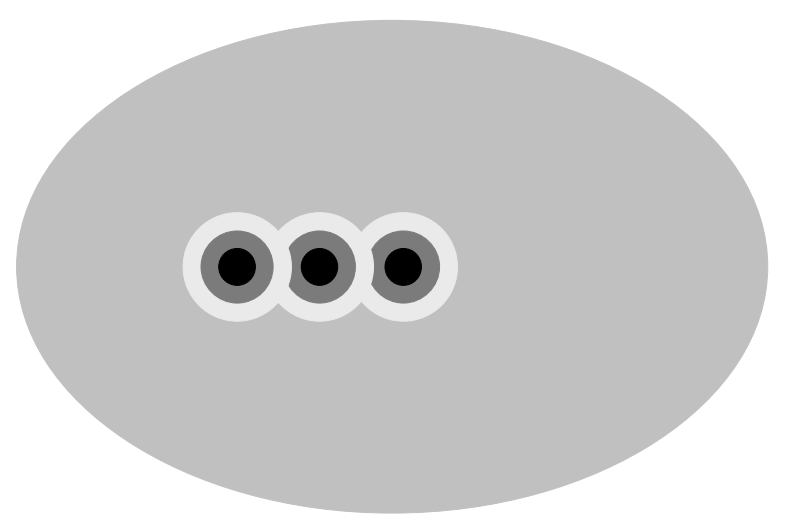

Fig. 2. Lateral target, off-axis evaluation. Sphere targets of three sizes $(2,4$, and $6 \mathrm{~cm}$ diameter) were placed at three locations (center of the patient, $3 \mathrm{~cm}$ lateral, and $6 \mathrm{~cm}$ lateral) for a total of 9 targets. mode. The ratio of BOT for FFF to flattened beams was calculated for each target.

\section{Mechanical rotation induced dosimetric uncertainty evaluation}

The effect of collimator and couch rotational uncertainty on plan quality in OAG was evaluated. Five representative patient plans were selected from SBRT patients with laterally located tumors treated at Mayo Clinic in Florida. The tumors ranged from 5.1 to $9.7 \mathrm{~cm}$ in lateral distance from the midline of the patient to the center of the tumor. Each case was planned with Pinnacle software using 6FFF for a Varian TrueBeam. For each of the five cases, a plan with a single full arc rotation was generated for OAG with $95 \%$ target volume in prescription dose (48 Gy in 4 fractions). This original plan was then copied and three new plans were generated with the following modifications: collimator rotation of one degree, couch rotation of one degree, and both collimator and couch rotations of one degree. One degree was chosen as a conservatively large estimate of rotation errors. The same MU settings were used for all plans. The same procedure was then repeated for CAG with the same prescription dose and normalization; however, only a partial arc was used. A full 360 degree arc rotation would not be deliverable with CAG because of a collision of the gantry with either the patient or treatment couch due to the lateral position of the isocenter. A total of eight plans were generated for each of the five cases.

Each plan was evaluated for planning target volume coverage, dose conformality, and critical organ dose. The target coverage was evaluated by the prescription isodose surface coverage (percent of the target volume receiving the prescription dose) for each of the plans. Conformality indices investigated were CI-100 and CI-50. The V20 of lung (percent of total normal lung receiving 20 Gy or more) was also determined.

\section{Statistical analysis}

For the systematic study, the results for the nine sphere targets are summarized descriptively. For the dosimetric mechanical uncertainty evaluation, we calculated absolute differences (ie, the absolute value of the difference) from the original plan for one degree collimator rotation, one degree couch rotation, and both collimator and couch rotations of one degree. These 
absolute differences were calculated separately for OAG and CAG for: prescription isodose surface coverage, CI-100, CI-50, and lung V20. The absolute differences from the original plan were compared between OAG and CAG using a paired t-test. $\mathrm{P}$-values of 0.05 or lower were considered statistically significant. Statistical analyses were performed using $\mathrm{R}$ Statistical Software (version 2.14.0; $\mathrm{R}$ Foundation for Statistical Computing, Vienna, Austria).

\section{Results and Discussion}

\section{Lateral target, off-axis geometry evaluation}

Results are shown in Table 1. 6FFF HI values were similar to $6 \mathrm{X}$ for all targets. 10FFF showed consistently worse HI values than $10 \mathrm{X}$ for all targets. Though differences in $\mathrm{HI}$ values were larger for $10 \mathrm{MV}$ beams than for $6 \mathrm{MV}$ beams, $10 \mathrm{MV}$ did not show considerable difference between flattened and unflattened beams, with the maximum $\mathrm{HI}$ difference being 0.10 . 6FFF CI-100 values were similar to $6 \mathrm{X}$ for both CAG and

Table 1a. Lateral target, off-axis geometry evaluation results for $6 \mathrm{MV}$.

\begin{tabular}{|c|c|c|c|c|c|c|c|}
\hline & \multirow{2}{*}{$\begin{array}{l}\text { Target Diameter } \\
\qquad(\mathrm{cm})\end{array}$} & \multicolumn{2}{|c|}{ Axis } & \multicolumn{2}{|c|}{$3 \mathrm{~cm}$ Off-Axis } & \multicolumn{2}{|c|}{$6 \mathrm{~cm}$ Off-Axis } \\
\hline & & Flattened & FFF & Flattened & FFF & Flattened & FFF \\
\hline \multirow[t]{3}{*}{$\mathrm{HI}$} & 2 & 1.19 & 1.17 & 1.16 & 1.15 & 1.14 & 1.13 \\
\hline & 4 & 1.11 & 1.11 & 1.11 & 1.12 & 1.12 & 1.10 \\
\hline & 6 & 1.11 & 1.13 & 1.11 & 1.13 & 1.12 & 1.11 \\
\hline \multirow[t]{3}{*}{ CI-100 } & 2 & 1.11 & 1.11 & 1.06 & 1.06 & 1.04 & 1.04 \\
\hline & 4 & 0.98 & 0.99 & 0.99 & 0.99 & 1.00 & 1.00 \\
\hline & 6 & 0.98 & 0.99 & 0.99 & 1.00 & 1.00 & 1.01 \\
\hline \multirow[t]{3}{*}{ CI-50 } & 2 & 4.23 & 4.12 & 4.33 & 4.28 & 4.33 & 4.30 \\
\hline & 4 & 3.36 & 3.36 & 3.36 & 3.29 & 3.39 & 3.38 \\
\hline & 6 & 3.11 & 3.17 & 3.17 & 3.17 & 3.21 & 3.24 \\
\hline \multirow[t]{3}{*}{ BOT ratio } & 2 & & 0.46 & & 0.47 & & 0.50 \\
\hline & 4 & & 0.47 & & 0.48 & & 0.50 \\
\hline & 6 & & 0.48 & & 0.49 & & 0.51 \\
\hline
\end{tabular}

HI: homogeneity index, CI: conformity index, BOT: beam on time. Note, FFF offers faster BOT than flattened beams.

Table 1b. Lateral target, off-axis geometry evaluation results for $10 \mathrm{MV}$.

\begin{tabular}{|c|c|c|c|c|c|c|c|}
\hline & \multirow{2}{*}{$\begin{array}{l}\text { Target Diameter } \\
\qquad(\mathrm{cm})\end{array}$} & \multicolumn{2}{|c|}{ Axis } & \multicolumn{2}{|c|}{$3 \mathrm{~cm}$ Off-Axis } & \multicolumn{2}{|c|}{$6 \mathrm{~cm}$ Off-Axis } \\
\hline & & Flattened & FFF & Flattened & FFF & Flattened & FFF \\
\hline \multirow[t]{3}{*}{$\mathrm{HI}$} & 2 & 1.23 & 1.25 & 1.20 & 1.21 & 1.17 & 1.19 \\
\hline & 4 & 1.13 & 1.17 & 1.14 & 1.19 & 1.14 & 1.17 \\
\hline & 6 & 1.12 & 1.20 & 1.13 & 1.23 & 1.13 & 1.21 \\
\hline \multirow[t]{3}{*}{ CI-100 } & 2 & 1.12 & 1.13 & 1.06 & 1.08 & 1.03 & 1.07 \\
\hline & 4 & 0.99 & 0.99 & 0.99 & 1.04 & 0.99 & 1.03 \\
\hline & 6 & 0.98 & 1.00 & 0.99 & 1.04 & 0.99 & 1.04 \\
\hline \multirow[t]{3}{*}{ CI-50 } & 2 & 4.59 & 4.49 & 4.73 & 4.64 & 4.71 & 4.65 \\
\hline & 4 & 3.48 & 3.40 & 3.54 & 3.53 & 3.56 & 3.52 \\
\hline & 6 & 3.13 & 3.12 & 3.24 & 3.29 & 3.25 & 3.29 \\
\hline \multirow[t]{3}{*}{ BOT ratio } & 2 & & 0.26 & & 0.27 & & 0.31 \\
\hline & 4 & & 0.27 & & 0.29 & & 0.32 \\
\hline & 6 & & 0.29 & & 0.30 & & 0.32 \\
\hline
\end{tabular}

HI: homogeneity index, CI: conformity index, BOT: beam on time. Note, FFF offers faster BOT than flattened beams. 
OAG for all target sizes. In contrast, CI-100 values were similar for $10 \mathrm{MV}$ flattened and unflattened beams in CAG, but for targets $3 \mathrm{~cm}$ OAG and $6 \mathrm{~cm}$ OAG, 10FFF CI-100 values were slightly worse compared to 10X. FFF beams gave better CI-50 values for $2 \mathrm{~cm}$ targets for 6 and $10 \mathrm{MV}$ in CAG and OAG. The advantage of FFF for CI-50 declined with increasing target diameter. Using FFF beams always reduced the BOT, as evidenced by ratios of BOT from $0.26-0.32$ for 10 MV and 0.46-0.51 for $6 \mathrm{MV}$. The benefit of reduced BOT with FFF decreased with off-axis distance of the target. The use of FFF beams with OAG increased the MU. This effect was greatest for the large targets at $6 \mathrm{~cm}$ off-axis. The max- imum MU increase for FFF to flattened beams was $19 \%$ for 6 $\mathrm{MV}$ and $28 \%$ for $10 \mathrm{MV}$.

\section{Mechanical rotation induced dosimetric uncertainty evaluation}

The isodose coverage, conformality, and V20 for all plans with mechanical rotation induced dosimetric uncertainty meet the requirements for RTOG protocols. Fig. 3 shows a representative axial slice for each case. As displayed in Table 2, the absolute difference in prescription isodose surface coverage from the original plan for collimator rotation, couch rotation, and collimator plus couch rotations of one degree ranged from
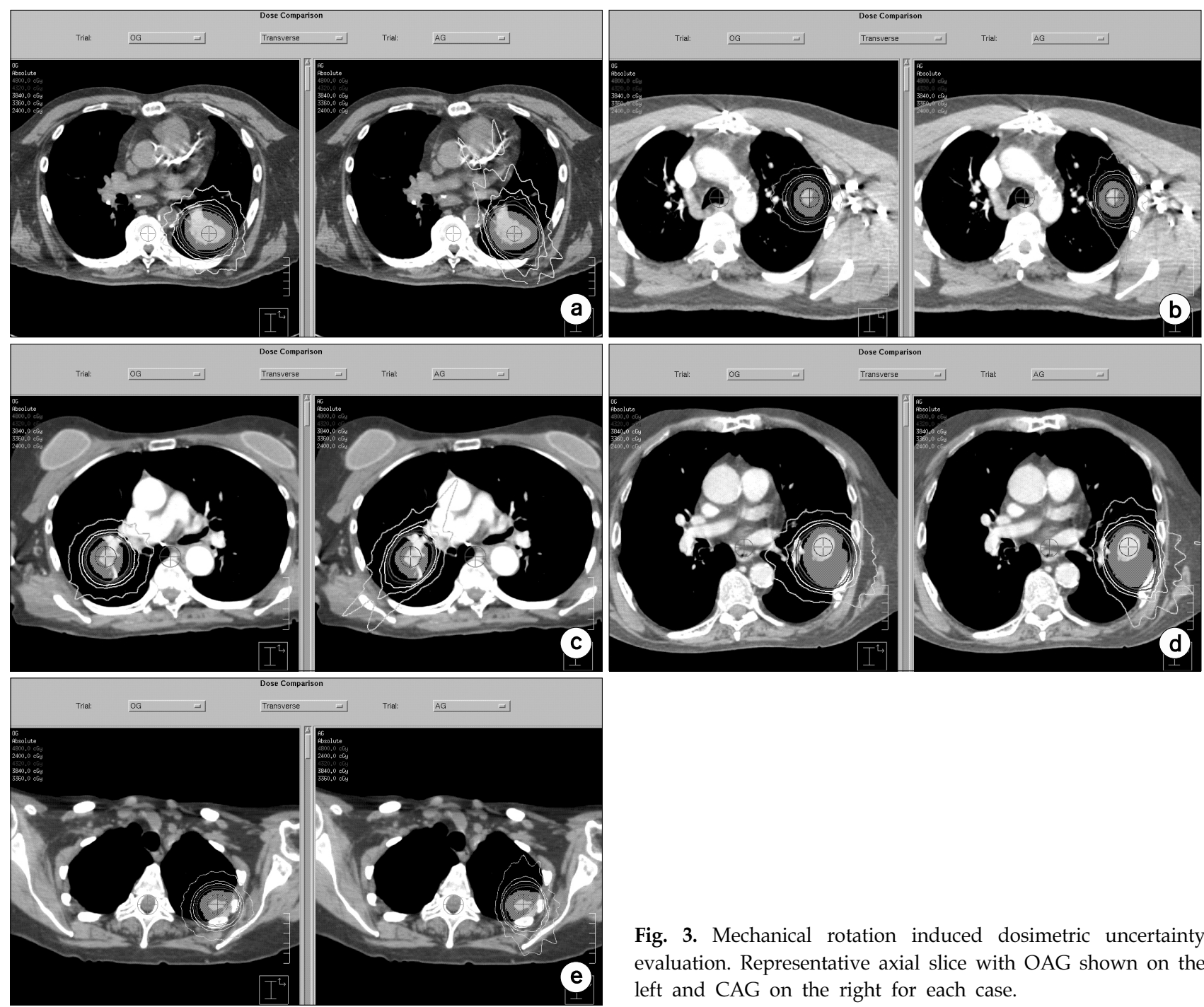

Fig. 3. Mechanical rotation induced dosimetric uncertainty evaluation. Representative axial slice with OAG shown on the left and CAG on the right for each case. 
PROGRESS in MEDICAL PHYSICS Vol. 25, No. 4, December, 2014

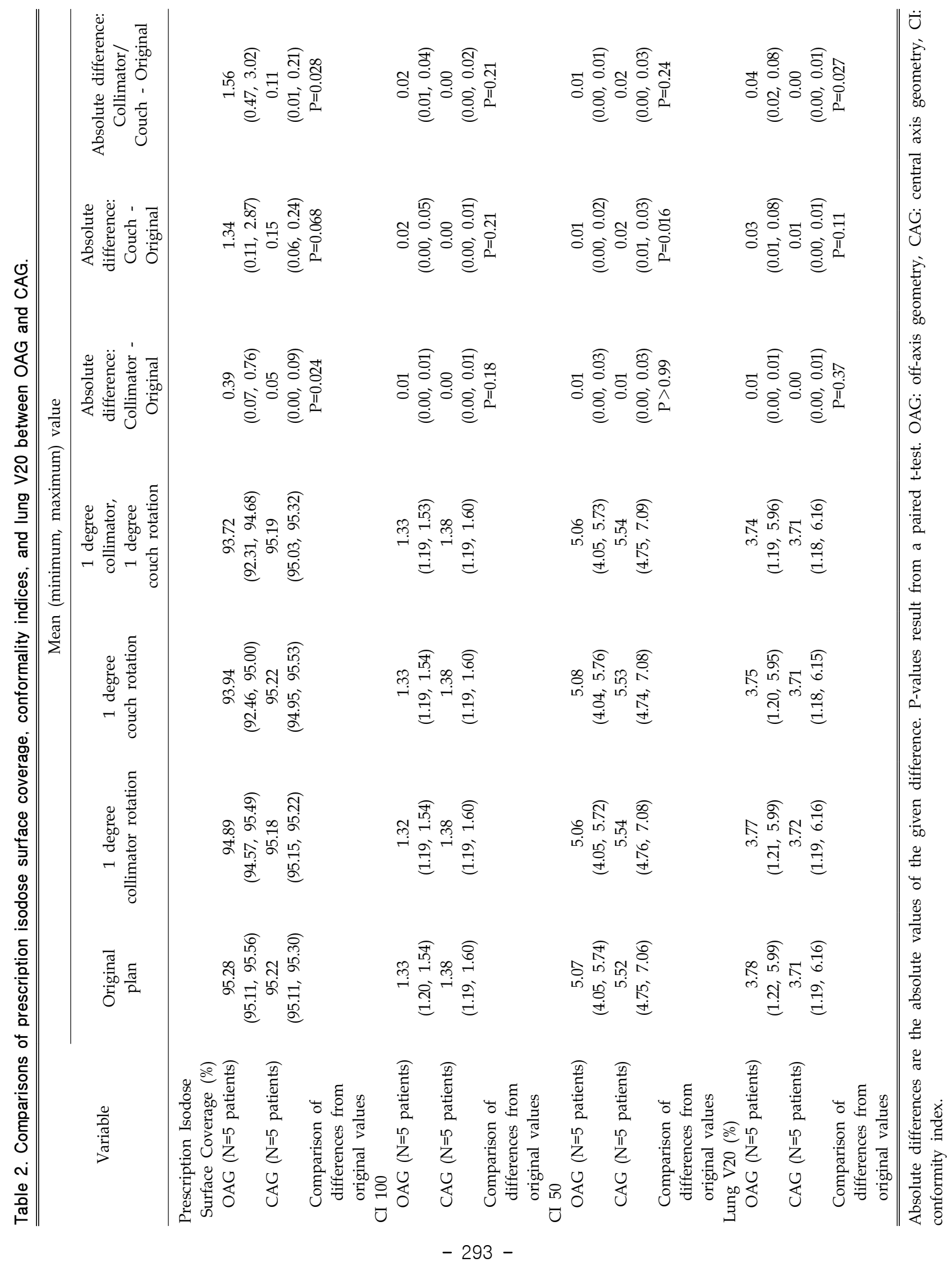


$0.10 \%$ to $3.00 \%$ for OAG and from $0.00 \%$ to $0.20 \%$ for CAG. In general, the effect of simulated couch or collimator rotation was to decrease the prescription isodose coverage. The variation from the original plan was significantly smaller for CAG compared to OAG for collimator rotation $(\mathrm{P}=0.024)$ and collimator plus couch rotation $(\mathrm{P}=0.028)$, with a similar, though not quite significant finding for couch rotation $(\mathrm{P}=0.068)$. Differences in prescription isodose surface coverage for each plan are further summarized in Fig. 4.

The conformality was evaluated for each plan. The absolute difference in CI-100 and CI-50 from original OAG and CAG plans for mechanical rotational error is shown in Table 2 . These absolute differences from baseline regarding collimator rotation, couch rotation, and collimator plus couch rotations were generally similar for CAG compared to OAG ( $\mathrm{P} \geq 0.18)$, with one small, but statistically significant difference occurring regarding $\mathrm{CI}-50$ for couch rotation $(\mathrm{P}=0.016)$. The con-

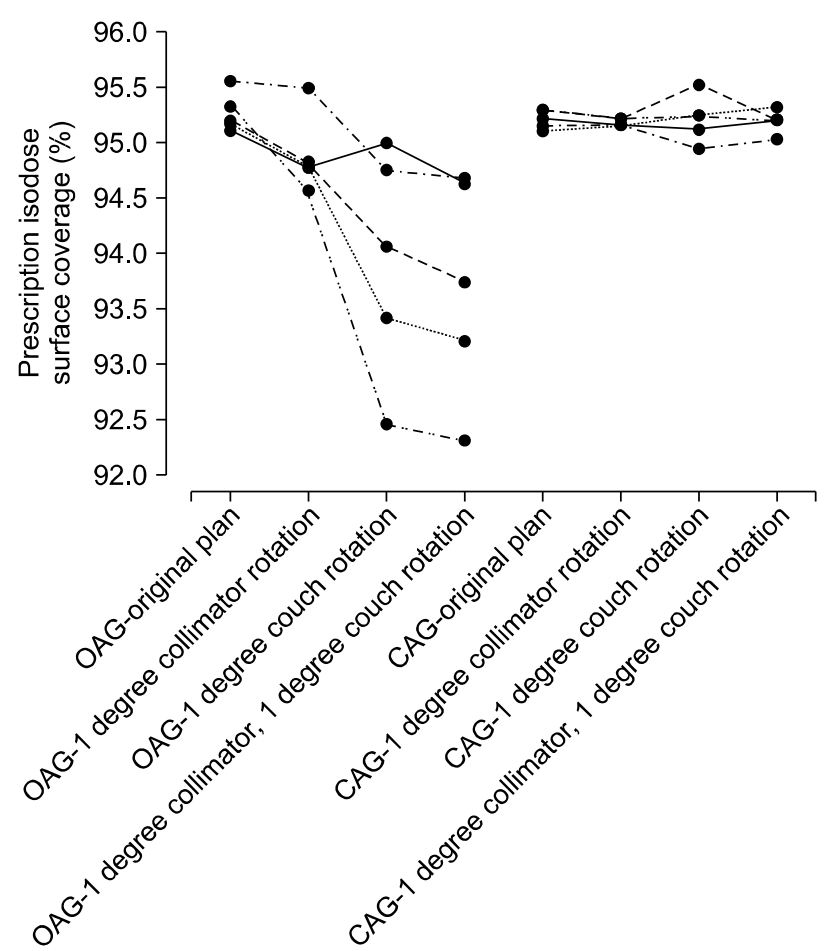

Fig. 4. Prescription Isodose Surface Coverage for off-axis geometry (OAG) and central axis geometry (CAG) for the original plan, one degree collimator rotation, one degree couch rotation, and one degree of both collimator and couch rotations. Points and lines for the same patient are shown in the same color. formality was found to increase or decrease depending on the shape of the target. CI-100 and CI-50 for OAG and CAG for collimator and couch rotational error are further illustrated in Fig. 5 and 6.

The V20 for normal lung was calculated for each plan. The mechanical rotation uncertainty improved the V20 in some cases, but caused it to be worse for other cases. The V20 was acceptable for all cases with all plans having a V20 less than $6 \%$. The absolute difference in V20 from the original OAG and CAG plans for collimator rotation, couch rotation, and collimator plus couch rotations are shown in Table 2 and Fig. 7. The variation from the original plan was relatively small for all cases; although it was smaller for CAG compared to OAG for collimator plus couch rotation $(\mathrm{P}=0.027)$.

\section{Discussion}

The results of this study demonstrate that $6 \mathrm{FFF}$ beams can

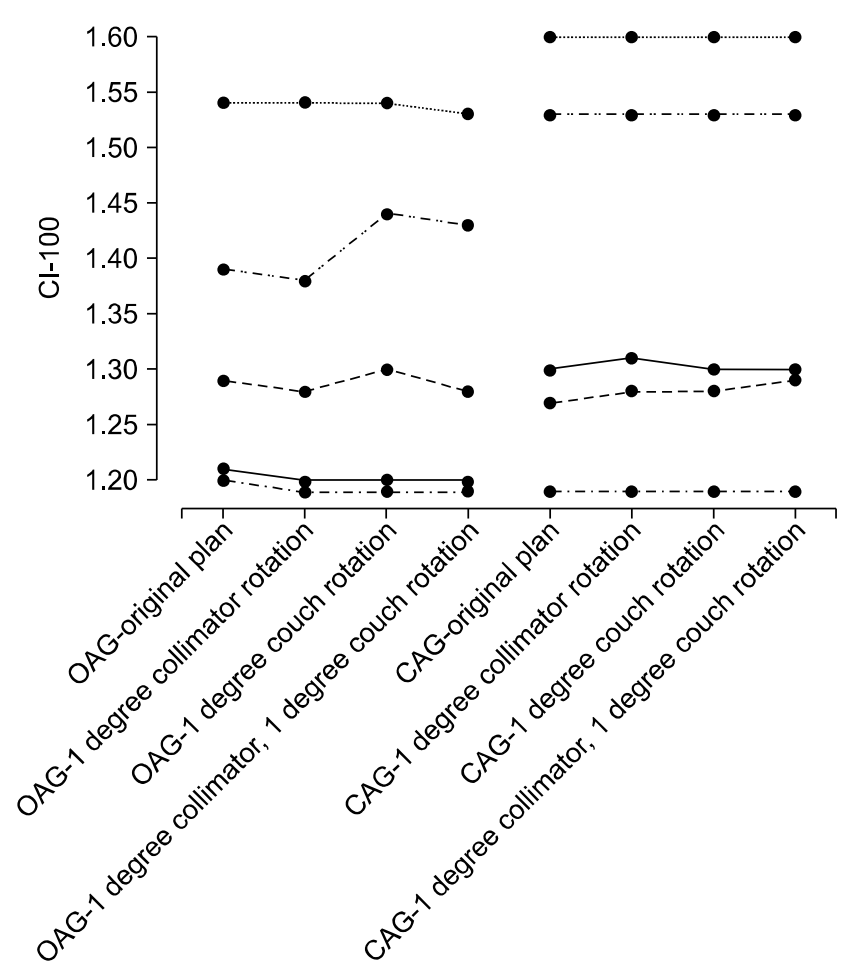

Fig. 5. CI-100 for off-axis geometry (OAG) and central axis geometry (CAG) for the original plan, one degree collimator rotation, one degree couch rotation, and one degree of both collimator and couch rotations. Points and lines for the same patient are shown in the same color. 


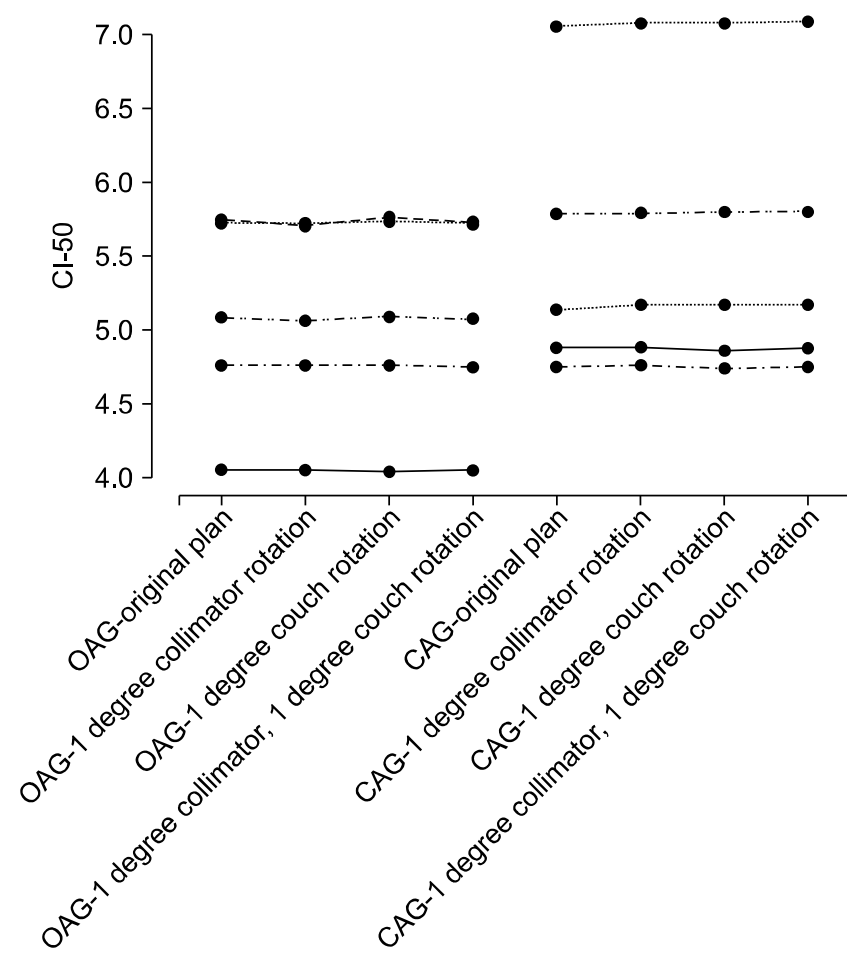

Fig. 6. CI-50 for off-axis geometry (OAG) and central axis geometry (CAG) for the original plan, one degree collimator rotation, one degree couch rotation, and one degree of both collimator and couch rotations. Points and lines for the same patient are shown in the same color.

be utilized in OAG without dosimetric compromise. 10FFF was shown to have less favorable $\mathrm{HI}$ values compared to $10 \mathrm{X}$, but the differences were small. For both 6 and $10 \mathrm{MV}$ beams, FFF provided a benefit for CI-50 for small targets in CAG and OAG. The advantage of FFF for CI-50 declined with target diameter. 10FFF can provide more dramatic BOT reduction compared to 6FFF; however, the benefit decreases with target off-axis distance when treating with OAG.

The mechanical rotation induced dosimetric variation was less for CAG compared to OAG, with some indices showing statistical significance, as seen in Table 2. Regardless, the differences were small in both CAG and OAG, and may not be clinically significant. The target isodose coverage decreased by an average of $1.00 \%$ for OAG when rotation error was present and by an average of $0.00 \%$ for CAG. The maximum absolute difference caused by rotational error was 0.05 and 0.08 for conformality indices and lung V20, respectively. All lung V20 results in this study met the RTOG dose objectives of less

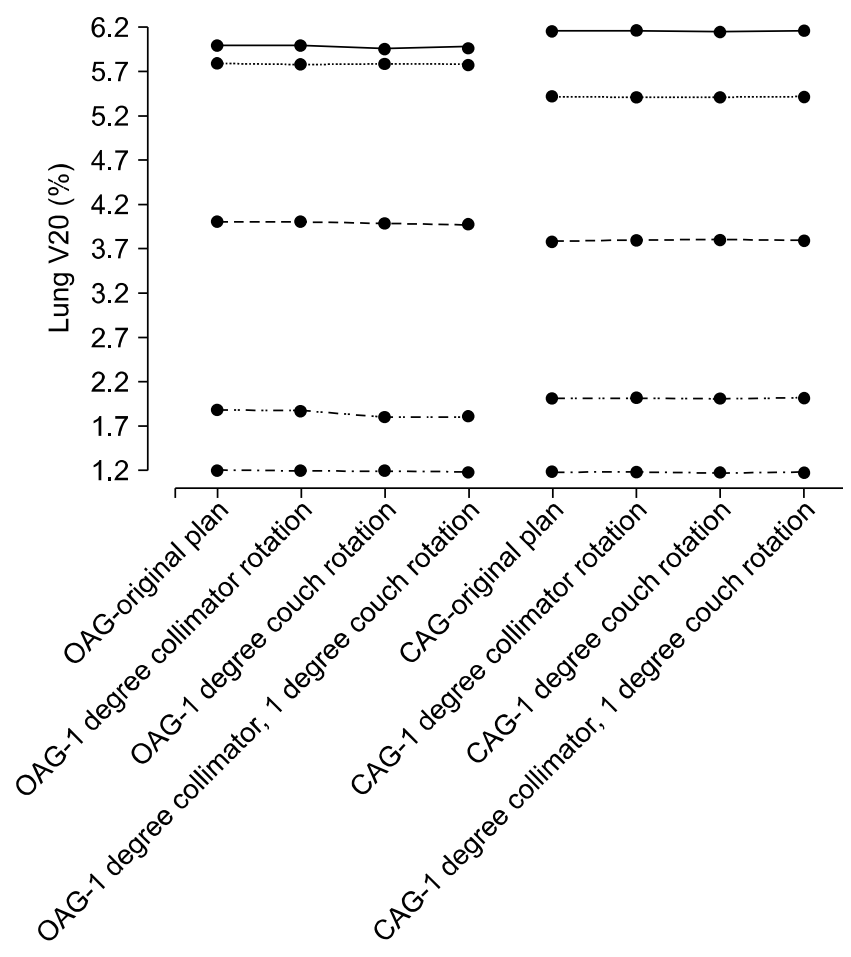

Fig. 7. Lung V20 for off-axis geometry (OAG) and central axis geometry (CAG) for the original plan, one degree collimator rotation, one degree couch rotation, and one degree of both collimator and couch rotations. Points and lines for the same patient are shown in the same color.

than $10 \%$. It should be noted that this study investigated the effects of one fraction. If additional fractions are used and the rotational error is random, then the dosimetric variation would be reduced.

The collimator and couch rotation investigated in this study was 1 degree, which is a conservatively large estimate to what may be expected in clinical practice. The tolerance for collimator or couch rotation is 0.5 degree. At our institution, annual quality assurance of the TrueBeam has demonstrated less than 0.3 degree and 0.2 degree for collimator and couch angle indicators, respectively. Therefore, the dosimetric variation due to rotation error found in this study is conservatively large.

While this study found that the effect of mechanical rotation error with OAG is acceptable, patient rotation during treatment could have an effect on the delivered dose distribution when treating in OAG. Josipovic et al investigated intra- and interfractional errors in patient position of SBRT and found with correction of translational errors the remaining rotational errors 
were approximately 1 degree. $^{18)}$ It is important in SBRT to correct patient oriented errors using image guidance techniques. Use of daily image guided radiation therapy (IGRT) is necessary to ensure accurate patient alignment and is part of the ACR-ASTRO practice guidelines for SBRT. ${ }^{19)}$

To evaluate which technique gave better dosimetric results, original CAG and OAG plans with no collimator or couch rotation can be compared (Table 2 and Fig. 3). This demonstrates OAG gave better dosimetric results for some cases, but not for others. The dosimetric results depend on the length of the arc rotation in relation to the tumor shape. For example, OAG with a full arc rotation gave a better dose distribution than CAG for spherical targets. CAG with a partial arc rotation was better than OAG for elliptical targets when the long axis of the target was in the direction of the arc. Both of these effects are more pronounced for low dose, as seen with CI-50. It should be noted that this comparison is influenced by the effect of using a full arc rotation in OAG and a partial arc rotation in CAG. While this is not a fair comparison, it does represent the clinical situation.

This study found that FFF beams in OAG can provide acceptable dose distributions. There are benefits and limitations to OAG and CAG. The selection of which to use when treating a laterally located target with FFF will depend on the situation and it may be influenced by whether a full or partial arc is desirable. This study showed an advantage of using OAG for small, spherical targets, which are often seen in SBRT. OAG is also beneficial for IGRT where a full gantry rotation is needed for cone-beam CT. However, it can also be argued that CAG is advantageous for IGRT because it is desirable to place the isocenter at the location of the target for imaging. Other benefits of CAG include reduced MU and less BOT. In some cases the choice to use OAG or CAG may depend on critical organs. Using a partial arc may reduce critical organ dose. It is also possible that a partial arc may be preferred over a full arc due to the possibility of retreatment, especially if SBRT is repeated for additional lesions.

\section{Conclusion}

6FFF can be used in OAG without dosimetric compromise. $10 \mathrm{FFF}$ provides more BOT reduction than 6FFF; however, it provides less favorable dosimetric indices compared to $6 \mathrm{FFF}$ in OAG. The benefit of reduced BOT with FFF decreases with distance of the target off-axis for both 6 and $10 \mathrm{MV}$. Mechanical error in collimator and couch rotation produces increased dosimetric variation for OAG compared to CAG; however, the variations in dose distributions for either treatment technique were small and may not be clinically significant. This study found that FFF beams can provide acceptable dose distributions in OAG.

\section{Competing Interests}

Siyong Kim owns interests of Medical Tool and Technology, LLC. The other authors have no interests to disclose.

\section{References}

1. Timmerman R, Galvin J, Michalski J, Straube W, lbbott G, Martin E, Abdulrahman R, Swann S, Fowler J, Choy $\mathrm{H}$ : Accreditation and quality assurance for Radiation Therapy Oncology Group: Multicenter clinical trials using Stereotactic Body Radiation Therapy in lung cancer. Acta Oncol 2006, 45(7):779-786.

2. Takeda A, Kunieda E, Sanuki N, Ohashi T, Oku Y, Sudo Y, Iwashita H, Ooka Y, Aoki Y, Shigematsu N, Kubo A: Dose distribution analysis in stereotactic body radiotherapy using dynamic conformal multiple arc therapy. Int $J$ Radiat Oncol Biol Phys 2009, 74(2):363-369.

3. Cashmore J: The characterization of unflattened photon beams from a $6 \mathrm{MV}$ linear accelerator. Phys Med Biol 2008, 53(7):1933-1946.

4. Stathakis S, Esquivel C, Gutierrez A, Buckey CR, Papanikolaou N: Treatment planning and delivery of IMRT using 6 and 18MV photon beams without flattening filter. Appl Radiat Isot 2009, 67(9):1629-1637.

5. Georg D, Knoos T, McClean B: Current status and future perspective of flattening filter free photon beams. Med Phys 2011, 38(3):1280-1293.

6. Kragl G, Baier F, Lutz S, Albrich D, Dalaryd M, Kroupa B, Wiezorek T, Knoos T, Georg D: Flattening filter free beams in SBRT and IMRT: dosimetric assessment of peripheral doses. Z Med Phys 2011, 21(2):91-101.

7. Thomas EM, Popple RA, Prendergast BM, Clark GM, Dobelbower MC, Fiveash JB: Effects of flattening filter-free and volumetric-modulated arc therapy delivery on treatment efficiency. J Appl Clin Med Phys 2013, 14(6):4328.

8. Kretschmer M, Sabatino M, Blechschmidt A, Heyden S, Grunberg B, Wurschmidt F: The impact of flattening-filter-free beam technology on 3D conformal RT. Radiat Oncol 2013, 8:133. 
9. Ross CC, Kim JJ, Chen ZJ, Grew DJ, Chang BW, Decker RH: A novel modified dynamic conformal arc technique for treatment of peripheral lung tumors using stereotactic body radiation therapy. Pract Radiat Oncol 2011, 1(2):126-134.

10. Shi C, Tazi A, Fang DX, lannuzzi C: Implementation and evaluation of modified dynamic conformal arc (MDCA) technique for lung SBRT patients following RTOG protocols. Med Dosim 2013, 38(3):287-290.

11. Kragl G, af Wetterstedt $S$, Knausl B, Lind $M$, McCavana P, Knoos T, McClean B, Georg D: Dosimetric characteristics of 6 and $10 \mathrm{MV}$ unflattened photon beams. Radiother Oncol 2009, 93(1):141-146.

12. Ponisch F, Titt U, Vassiliev ON, Kry SF, Mohan R: Properties of unflattened photon beams shaped by a multileaf collimator. Med Phys 2006, 33(6):1738-1746.

13. Yarahmadi M, Allahverdi M, Nedaie HA, Asnaashari K, Vaezzadeh SA, Sauer OA: Improvement of the penumbra for small radiosurgical fields using flattening filter free low megavoltage beams. Z Med Phys 2013, 23(4):291-299.

14. Kim S: UF image-guided stereotactic body radiation therapy (IGSBRT) program for lung treatment using Elekta Synergy. In
IFMBE Proceedings. Edited by Magjarevic R. Springer; 2007.

15. Olivier K, Chung $\mathrm{H}$, Jin $\mathrm{H}$, Yang $\mathrm{H}$, Kim S: Intra-Fraction Patient Displacement During Image Guided Stereotactic Body Radiation Therapy (IGSBRT) for Lung Treatment. Int J Radiat Oncol Biol Phys 2006, 66(3):S489.

16. Khoo VS, Oldham M, Adams EJ, Bedford JL, Webb S, Brada M: Comparison of intensity-modulated tomotherapy with stereotactically guided conformal radiotherapy for brain tumors. Int J Radiat Oncol Biol Phys 1999, 45(2):415-425.

17. Murphy MJ, Chang S, Gibbs I, Le QT, Martin D, Kim $D$ : Image-guided radiosurgery in the treatment of spinal metastases. Neurosurg Focus 2001, 11(6):e6.

18. Josipovic M, Persson GF, Logadottir A, Smulders B, Westmann G, Bangsgaard JP: Translational and rotational intra- and inter-fractional errors in patient and target position during a short course of frameless stereotactic body radiotherapy. Acta Oncol 2012, 51(5):610-617.

19. ACR-ASTRO practice guideline for the performance of stereotactic body radiation therapy. Practice Guidelines and Technical Standards. Reston, VA: American College of Radiology; 2009. 